\title{
A Retrospective Claims Analysis of Advanced Prostate Cancer Costs and Resource Use
}

\author{
Sreevalsa Appukkuttan ${ }^{1} \cdot$ Krishna Tangirala $^{1} \cdot$ Svetlana Babajanyan $^{1} \cdot$ Lonnie Wen $^{1} \cdot$ Stacey Simmons $^{1} \cdot$ Neal Shore $^{2}$
}

Published online: 22 October 2019

(c) The Author(s) 2019

\begin{abstract}
Background Castration-resistant prostate cancer (CRPC) is associated with high costs and healthcare resource utilization (HCRU). Objective This study followed patients with CRPC through their continuum of care and analyzed claims data regarding treatments, total HCRU, and costs, both before and after metastasis diagnosis.

Methods A retrospective cohort of patients with newly diagnosed metastatic CRPC (mCRPC) in the USA was identified from the Truven Health MarketScan database from January 2009 to March 2015. The mCRPC algorithm employed International Classification of Diseases, Ninth Revision codes for prostate cancer (pre-index) and secondary metastatic disease (index date) and a subsequent claim for a US FDA-approved treatment for mCRPC. Patient inclusion required evidence of surgical or pharmacological castration and no evidence of bone-targeted treatments during the baseline period while evaluating continuous enrollment 25 months pre-index and 6 months post-index. Treatment patterns were assessed during pre- and postindex periods; HCRU and costs were annualized for comparison purposes regarding both pre- and post-index timeframes.

Results Among 261 patients with mCRPC (mean age 72 years), the most common treatments during the pre-index period were bicalutamide (90.04\%), leuprolide (81.99\%), abiraterone (22.22\%), docetaxel (20.69\%), and ketoconazole $(18.01 \%)$. Mean per-patient-per-year (PPPY) all-cause annualized healthcare costs significantly increased from $\$$ US35,102.55 in the pre-index nonmetastatic CRPC (nmCRPC) period to \$US156,499.89 after metastasis diagnosis (mCRPC). Mean PPPY inpatient admissions and emergency department visits increased from 0.20 to 1.36 and from 0.63 to 1.56 , respectively.

Conclusions Average yearly costs and HCRU were four times higher following mCRPC diagnosis, indicating a need for appropriate management strategies to optimize the potential delay of disease progression among patients with nmCRPC.
\end{abstract}

\section{Key Points for Decision Makers}

Treatment of patients with metastatic castration-resistant prostate cancer (mCRPC), the most aggressive clinical state of prostate cancer, represents a significant burden on the healthcare system. Both healthcare resource utilization (HCRU) and costs are higher in the mCRPC setting than in the nonmetastatic (nmCRPC) setting.

Improvements in the care and management of patients with nmCRPC may mitigate a portion of this healthcare burden and subsequent cost of care.

Sreevalsa Appukkuttan

sreevalsa.appukkuttan@bayer.com

1 Bayer US, 100 Bayer Blvd, Whippany, NJ 07981, USA

2 Carolina Urologic Research Center, Atlantic Urology Clinics, Myrtle Beach, SC 29572, USA

\section{Introduction}

Prostate cancer is the second-most common cancer among men in the USA, with an estimated 164,690 new cases and 29,430 deaths in 2018 [1]. Early growth of prostate cancer is the result of androgen receptor stimulation by testosterone, but tumor proliferation becomes increasingly independent of testosterone signaling as the disease progresses [2].

An estimated 10-20\% of men will develop castrationresistant prostate cancer (CRPC) within 5 years after their prostate cancer diagnosis [3]. CRPC represents an advanced form of prostate cancer characterized by disease progression following appropriate surgical or pharmacologic castration in the form of androgen-deprivation therapy (ADT) [3]. CRPC can occur with or without associated metastases [i.e., nonmetastatic (nmCRPC) vs. metastatic CRPC (mCRPC)], although nmCRPC will eventually progress to $\mathrm{mCRPC}$, with greater morbidity and mortality associated with this stage of the disease [3]. 
Until recently, patients with nmCRPC were managed with continued ADT and occasionally second-line interventions (first-generation androgen-receptor inhibitors, ketoconazole, estrogens, steroids), none of which had demonstrated level one evidence for either a survival benefit or significant delay in radiographic progression to mCRPC. Apalutamide, enzalutamide, and darolutamide have been evaluated in placebo-controlled trials in patients with high-risk nmCRPC, generally defined as a prostate-specific antigen doubling time of $\leq 10$ months, a time period shown to indicate aggressive disease likely to metastasize [4-6]. In 2018, apalutamide (Erleada ${ }^{\circledR}$ ) and enzalutamide $\left(\mathrm{Xtandi}^{\circledR}\right)$ were approved by the US FDA for the treatment of nmCRPC based on their ability to significantly prolong metastases-free survival (i.e., delay the time to diagnosis of mCRPC or death) versus continued ADT alone.

The healthcare economic burden of prostate cancer can be substantial. The direct medical cost of prostate cancer was estimated to be the fifth largest cancer-related economic burden in the USA in 2010 (at \$US11.9 billion) and is projected, based on assumptions of future cancer incidence, survival, and increases in cost of care, to be the third largest, at \$US16.3 billion in 2020, primarily due to expected increases in survival and the expansion of an aging population [7]. Studies have shown that healthcare resource utilization (HCRU) and costs increase with progressive and metastatic prostate cancer [8-10].

However, evidence describing HCRU and costs associated with nmCRPC versus mCRPC or comparing HCRU and costs before and after diagnosis of mCRPC is limited. One challenge to assessing the economic burden of nmCRPC using claims data is that hormone-sensitive prostate cancer and CRPC (including nmCRPC and mCRPC) cannot be differentiated using International Classification of Diseases (ICD) coding alone. A few studies have attempted to define algorithms to identify patients with CRPC and those with mCRPC using claims data $[8,11$, 12], but each have faced limitations, including lack of complete medical, laboratory, and pharmaceutical claims data or inclusion of a homogenous population of privately insured patients only. One study used a combination of International Classification of Diseases, Ninth Revision, Clinical Modification (ICD-9-CM) codes and prescription claims for mCRPC drugs to identify patients with mCRPC [12]. The present study similarly used this combination and also considered evidence of castration and a premetastatic diagnosis index period in an attempt to identify patients with CRPC before and after the diagnosis of metastases.

This study was a retrospective review of claims data that aimed to assess how costs and HCRU changed as prostate cancer progressed to metastatic disease by examining the annualized HCRU and costs associated with both nmCRPC and mCRPC. This information may be useful in informing economic models as future treatments of nmCRPC are investigated.

\section{Methods}

\subsection{Data Source and Study Design}

A pre- and post-retrospective cohort study was conducted in the USA using administrative claims data from a large, commercially insured US population available from two databases: the Truven Health Analytics MarketScan ${ }^{\circledR}$ Commercial Claims and Encounters (CCAE) and Truven Health Analytics MarketScan ${ }^{\circledR}$ Medicare Supplemental and Coordination of Benefits (COB) database. The CCAE database includes individuals aged 18-64 years and contains information on demographics, enrollment, inpatient and outpatient services, outpatient prescription drug use, and carve-out services (e.g., mental health services) from approximately 100 large employers, health plans, and government and public organizations. The Medicare database covers individuals aged $\geq 65$ years who are Medicare-eligible retirees with employer-sponsored Medicare supplemental plans. Both databases are fully compliant with the Health Insurance Portability and Accountability Act of 1996 in relation to the use of deidentified patient data [13].

Claims from the CCAE and Medicare COB databases were used to identify patients with CRPC who developed mCRPC during the study period. Patients served as their own controls and were evaluated in pre-progression (nmCRPC) and post-progression (mCRPC) phases. The index date was defined as the date of the first claim with a diagnosis of metastases. Treatment utilization was assessed in the 25 months prior to mCRPC diagnosis (pre-index period/baseline), which is the median time reported in the literature from the development of castration resistance to the development of bone metastases [14]. HCRU and costs were annualized from the 6 months post-index data. The study design is shown in Fig. 1.

\subsection{Patient Population}

The study population included all patients with prostate cancer with incident mCRPC between 1 January 2009 and 31 March 2015 (index identification period) with the date of first diagnosis of metastatic disease serving as the index diagnosis date. Eligible patients were identified using the following inclusion criteria:

(a) At least one diagnosis of prostate cancer (ICD-9-CM code 185 , malignant neoplasm of prostate) on one or 
Fig. 1 Study design. HCRU healthcare resource utilization
STUDY PERIOD: DECEMBER 2006 - SEPTEMBER 30, 2015

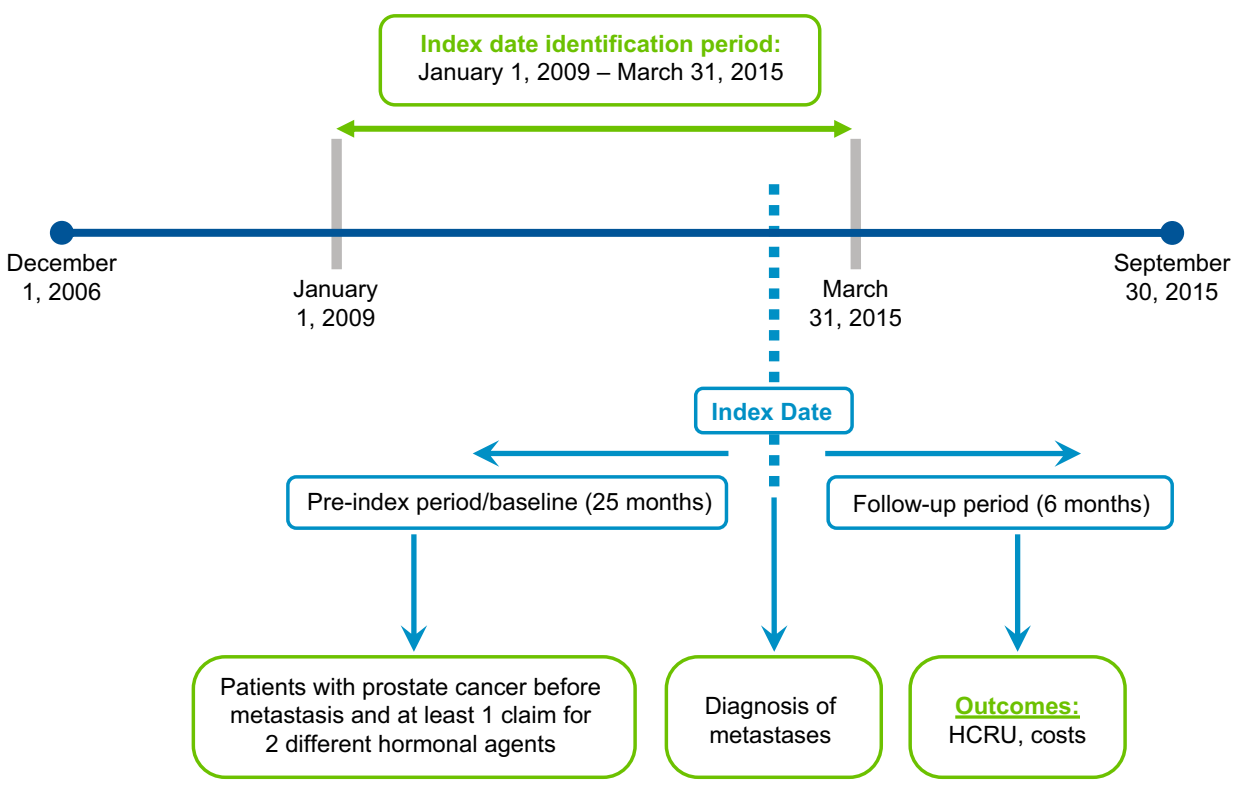

more inpatient claim or two or more outpatient claims (at least 30 days apart) during the index period.

(b) Evidence of surgical castration (based on current procedural terminology codes 54530, 54533, 54540, 54535, 54690; ICD-9-CM procedure codes 62.3, 62.41, 62.42; or ICD-9-CM diagnosis code V45.77) or medical castration (defined as having one or more claim for any of the anti-androgens, androgen-synthesis inhibitors, estrogens, and progestins and one claim for any of the luteinizing hormone-releasing hormone (LHRH) agonists).

(c) Diagnosis of metastasis (ICD-9-CM code 196.0-196.9, 197.0-197.8, 198.5, a secondary malignant neoplasm of lymph nodes, visceral organs, and/or bone/bone marrow) on one or more inpatient claim or two or more outpatient claims (at least 30 days apart) during the index period.

(d) At least one claim of FDA-approved treatment for mCRPC (docetaxel, cabazitaxel, abiraterone, enzalutamide, radium-223, mitoxantrone, estramustine, and sipuleucel-T) within 3 months of diagnosis of metastatic disease.

(e) Continuous enrollment in a health plan for at least 25 months prior to the index date (to ensure adequate time for castrate resistance to develop) and 6 months after the index date.

Patients were excluded if they had a claim for a non-prostate cancer or non-skin cancer diagnosis in the 25-month baseline period (to ensure prostate cancer was the primary diagnosis), if they were female, if they were aged $<18$ years on the index date, or if they had negative cost claims in the study period. Patients with evidence of bone treatments, including receipt of zoledronic acid or denosumab, in the 6-month pre-index period (i.e., prior to diagnosis of metastases) were also excluded to ensure patients were nonmetastatic in the baseline period.

\subsection{Study Variables and Outcomes}

Demographic and baseline clinical characteristics were recorded on the index date and included age, plan type, plan region, castration type, metastases type, and Klabunde's adaptation for prostate cancer of the Charlson Comorbidity Index score, which incorporates both physician and inpatient claims data and is validated in patients with prostate cancer [15]. Higher scores are indicative of a greater number of comorbid conditions and reduced survival [15].

The main outcomes included treatments received in the 25-month pre-index period and 6-month post-index period, annualized HCRU, and costs pre- and post-index. Medical and pharmacy claims were used to determine HCRU and costs. HCRU included the number of visits by setting (emergency department (ED), inpatient, office visits, and outpatient services), length of hospital stay among those with inpatient visits, and number of pharmacy claims. Healthcare costs included costs of visits by site and costs of pharmacy claims.

\subsection{Statistical Analysis}

A descriptive analysis of the study sample was conducted using means \pm standard deviations for continuous variables 
and percentages for categorical variables. Healthcare cost was based on total reimbursed/paid charges for all HCRU, and costs were standardized to \$US, year 2015 values using the Consumer Price Index for US medical care. Costs were estimated for individual patients and summarized at the sample level. We also calculated per-patient per-month (PPPM) and per-patient per-year (PPPY) costs. The average monthly costs during the 6-month post-index period were annualized to determine costs during the 12-month post-index period. Annualized HCRU and costs in the pre- and post-index periods were compared using paired $t$ tests or Wilcoxon signedrank tests, depending on the distributional properties of the variables. A $P$ value of $<0.05$ was used to denote statistically significant differences.

\section{Results}

\subsection{Baseline Characteristics}

From a total of 388,290 patients with evidence of secondary metastasis ICD-9-CM codes, 9306 patients remained after continuous eligibility criteria and prostate cancer codes at baseline were applied (Fig. 2). From this cohort, after applying the baseline medical or surgical castration criteria

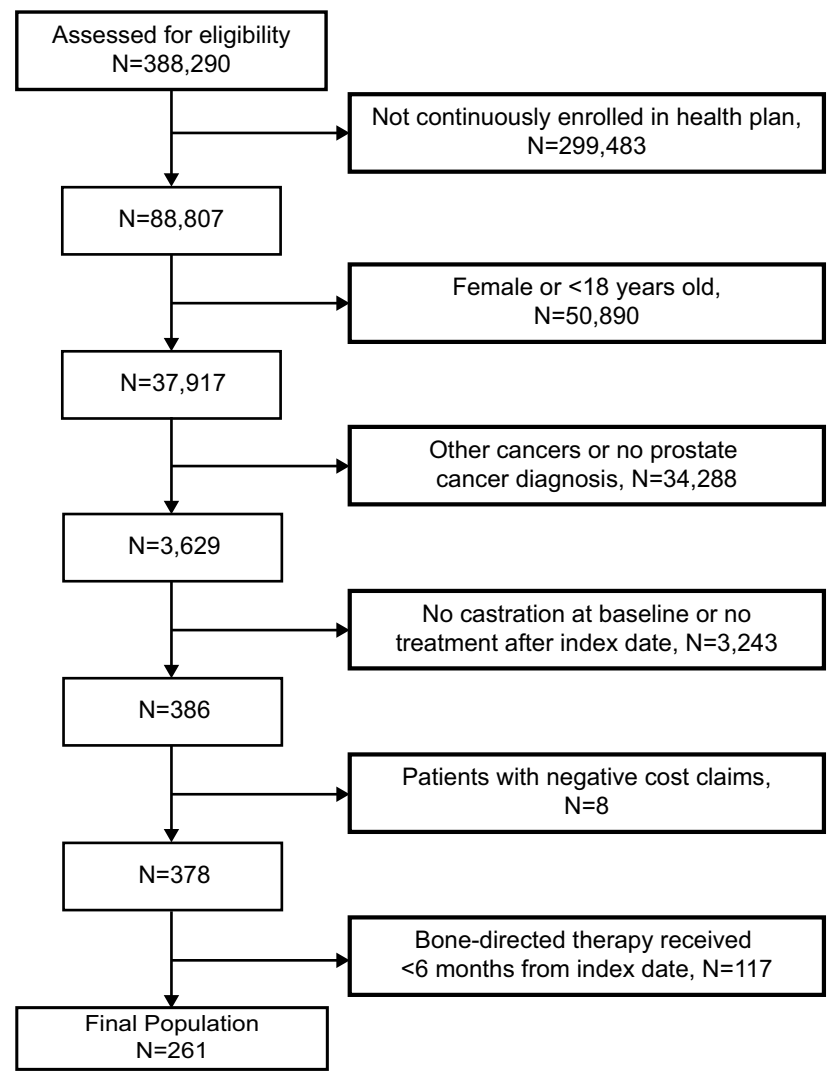

Fig. 2 CONSORT diagram and excluding those with bone treatments, 261 patients were available for analysis. Patient demographics and clinical characteristics are shown in Table 1 . The mean age at the index date was $72.11 \pm 10.39$ years. The majority of patients (70.5\%) had Medicare, and the most common plans were comprehensive or preferred provider organization/exclusive provider organization ( $42.15 \%$ and $36.02 \%$, respectively).

Table 1 Patient demographic and clinical characteristics at baseline

\begin{tabular}{|c|c|}
\hline Characteristic & Total $(N=261)$ \\
\hline Age (years) & $72.11 \pm 10.39$ \\
\hline \multicolumn{2}{|l|}{ Age group } \\
\hline$\leq 64$ & $76(29.12)$ \\
\hline $65-74$ & 73 (27.97) \\
\hline $75-84$ & $82(31.42)$ \\
\hline$\geq 85$ & $30(11.49)$ \\
\hline \multicolumn{2}{|l|}{ Plan type } \\
\hline Medicare & $184(70.50)$ \\
\hline Commercial & $77(29.50)$ \\
\hline \multicolumn{2}{|l|}{ Plan } \\
\hline Comprehensive & $110(42.15)$ \\
\hline PPO/EPO & $94(36.02)$ \\
\hline $\mathrm{HMO}$ & $26(9.96)$ \\
\hline POS/POS with capitation & $19(7.28)$ \\
\hline CDHP/HDHP & $7(2.68)$ \\
\hline Missing/unknown & $5(1.92)$ \\
\hline \multicolumn{2}{|l|}{ Region } \\
\hline Northeast & $41(15.71)$ \\
\hline North central & $89(34.10)$ \\
\hline South & $93(35.63)$ \\
\hline West & $38(14.56)$ \\
\hline Klabunde comorbidity score & $0.6 \pm 0.75$ \\
\hline \multicolumn{2}{|c|}{ Klabunde comorbidity categories } \\
\hline 0 & $100(38.31)$ \\
\hline 1 & $101(38.70)$ \\
\hline$>1$ & $60(22.99)$ \\
\hline \multicolumn{2}{|l|}{ Castration (baseline) } \\
\hline Medical & $260(99.62)$ \\
\hline Surgical & $1(0.38)$ \\
\hline Surgical and medical & $0(0)$ \\
\hline \multicolumn{2}{|l|}{ Metastases type } \\
\hline Bone only & $207(79.31)$ \\
\hline Visceral only & $16(6.13)$ \\
\hline Lymph only & $28(10.73)$ \\
\hline Bone and visceral only & $5(1.92)$ \\
\hline Bone and lymph only & $5(1.92)$ \\
\hline
\end{tabular}

Data are presented as mean \pm standard deviation or $N(\%)$ unless otherwise indicated

$C D H P$ consumer-driven health plan, $E P O$ exclusive provider organization, $H D H P$ high-deductible health plan, $H M O$ health maintenance organization, $P O S$ point of service, $P P O$ preferred provider organization, $S D$ standard deviation 
The mean Klabunde comorbidity score was $0.6 \pm 0.75$, and $22.99 \%$ of patients had scores $>1$. A vast majority of patients $(79.31 \%)$ had bone-only metastases.

\subsection{Treatment Utilization}

The 25 months leading up the diagnosis of mCRPC (i.e., pre-index period) included claims for at least 20 different drug treatments, including anti-androgens, LHRH agonists, LHRH antagonists, androgen-synthesis inhibitors, progestins, and chemotherapies. The most common treatments in the nmCRPC stage were bicalutamide (90.04\%), leuprolide (81.99\%), abiraterone (22.22\%), docetaxel (20.69\%), and ketoconazole $(18.01 \%)$. Conversely, the most common treatments after mCRPC were leuprolide (65.52\%), abiraterone (49.81\%), docetaxel (43.68\%), bicalutamide (23.75\%), and enzalutamide $(15.71 \%)$. The proportion of patients using bicalutamide, leuprolide, and ketoconazole treatments decreased once metastases were diagnosed compared with the nonmetastatic state (Fig. 3).

\subsection{Healthcare Resource Utilization and Costs}

The mean PPPY number of total medical and pharmacy claims for nmCRPC (pre-index period) and mCRPC (postindex period) were 63.79 and 119.95 , respectively. All-cause HCRU PPPY, including ED, inpatient, office, and outpatient visits, were all significantly higher (all, $P<0.05$ ) for patients in the mCRPC stage than for those in the nmCRPC stage. In addition to the increase in inpatient admissions, the mean total length of inpatient stay for patients with mCRPC was longer than for those with nmCRPC (6.80 vs. 0.94 days; $P<0.0001)$. The mean number of prescription claims was also significantly higher for mCRPC than for nmCRPC (50.05 vs. 31.12; $P<0.0001$ ) (Table 2 ).
Mean PPPY all-cause total medical and pharmacy costs were significantly higher (all, $P<0.05$ ) in patients with mCRPC than in those with nmCRPC ( $\$$ US156,499.89 vs. 35,102.55) (Table 2). Mean PPPY costs for outpatient services, which comprised the largest proportion of the total cost, increased from $\$$ US20,056.82 to $83,822.08$ $(P<0.0001)$. Pharmacy costs (representing oral medications) had the highest proportional increase of more than five times from the nmCRPC stage to the mCRPC stage (\$US7942.49 vs. $41,458.79 ; P<0.0001$ ).

Monthly healthcare costs in the 6 months pre- and postindex are shown in Fig. 4. Total PPPM medical and pharmacy costs peaked during the month of mCRPC diagnosis and remained substantially higher during the MCRPC stage in comparison with the nmCRPC stage. Notably, PPPM oral drug costs progressively increased after diagnosis of metastatic disease.

\section{Discussion}

Using a commercial claims database, this study found that annualized mean PPPY all-cause HCRU and costs for patients with CRPC were significantly higher after diagnosis of metastatic disease. Our findings show that costs began to increase during the month prior to metastases diagnosis, reaching a peak 1 month after diagnosis and then tapering down; however, total costs remained significantly higher after metastasis diagnosis compared with the nonmetastatic stage. These results also show the important drivers of increased costs and resource use in mCRPC, with mean pharmacy costs and inpatient admission costs increasing by more than fivefold and mean outpatient services costs increasing by more than fourfold.
Fig. 3 Frequency of treatment utilization. $m C R P C$ metastatic castration-resistant prostate cancer, $n m C R P C$ nonmetastatic castration-resistant prostate cancer

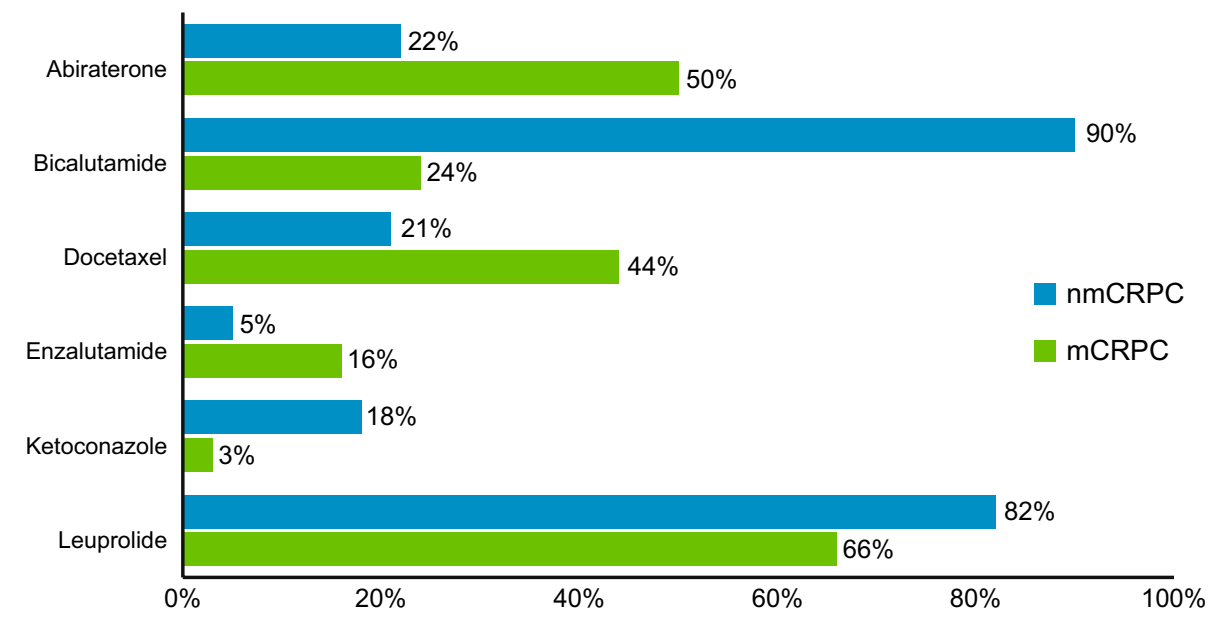

Key: mCRPC - metastatic castration-resistant prostate cancer; nmCRPC - nonmetastatic castration-resistant prostate cancer. 
Table 2 All-cause healthcare resource utilization and costs (per patient per year)

\begin{tabular}{|c|c|c|c|}
\hline Resources and costs & Pre-index period (nmCRPC) & Post-index period (mCRPC) & $P$ value \\
\hline \multicolumn{4}{|l|}{ Healthcare resource } \\
\hline Total medical and pharmacy claims & $63.79 \pm 31.45$ & $119.95 \pm 49.41$ & $<0.0001$ \\
\hline Pharmacy claims & $31.12 \pm 20.97$ & $50.05 \pm 26.39$ & $<0.0001$ \\
\hline Inpatient admissions & $0.20 \pm 0.36$ & $1.35 \pm 1.79$ & $<0.0001$ \\
\hline Office visits & $11.69 \pm 5.87$ & $21.03 \pm 10.28$ & $<0.0001$ \\
\hline ED visits & $0.63 \pm 1.55$ & $1.56 \pm 2.38$ & $<0.0001$ \\
\hline Outpatient services & $20.14 \pm 12.15$ & $45.95 \pm 28.03$ & $<0.0001$ \\
\hline Total length of inpatient stay (in days) & $0.94 \pm 2.58$ & $6.80 \pm 17.44$ & $<0.0001$ \\
\hline \multicolumn{4}{|l|}{ Healthcare costs } \\
\hline Total medical and pharmacy & $35,102.55 \pm 39,524.11$ & $156,499.89 \pm 105,581.14$ & $<0.0001$ \\
\hline Pharmacy & $7942.49 \pm 11,590.16$ & $41,458.79 \pm 38,327.74$ & $<0.0001$ \\
\hline Inpatient admissions & $5288.90 \pm 23,822.33$ & $26,749.69 \pm 62,366.92$ & $<0.0001$ \\
\hline Office visits & $1341.07 \pm 988.47$ & $2978.89 \pm 6442.89$ & $<0.0001$ \\
\hline ED visits & $473.27 \pm 1127.86$ & $1490.45 \pm 4717.63$ & 0.0005 \\
\hline Outpatient services & $20,056.82 \pm 24,039.01$ & $83,822.08 \pm 88,066.36$ & $<0.0001$ \\
\hline
\end{tabular}

Data are presented as mean \pm standard deviation unless otherwise indicated. Costs are standardized to \$US, year 2015 values

$E D$ emergency department, $m C R P C$ metastatic castration-resistant prostate cancer, $n m C R P C$ nonmetastatic castration-resistant prostate cancer

Fig. 4 Total monthly healthcare costs per patient. $m C R P C$ metastatic castration-resistant prostate cancer, $n m C R P C$ nonmetastatic castration-resistant prostate cancer, $R x$ prescription claims

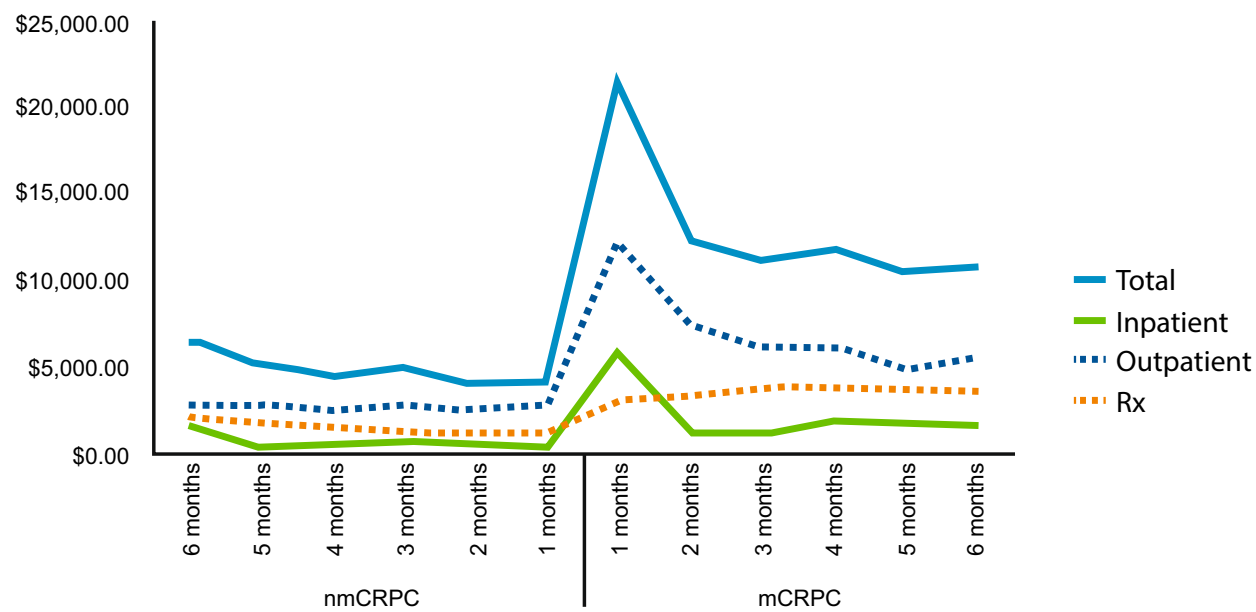

Key: mCRPC - metastatic castration-resistant prostate cancer; nmCRPC - nonmetastatic castration-resistant prostate cancer; Rx - prescription claims.
Our findings are consistent with research showing increased healthcare utilization and costs associated with the development of metastatic prostate cancer. In an observational cohort study of patients initially diagnosed with localized prostate cancer $(N=7482)$ [9], patients with subsequent metastases had substantially higher medical utilization and costs relative to a control sample $(N=25,709)$ without metastases. Over the 24-month observation period, medical costs for controls were relatively constant (weighted mean PPPM \$US2746; year 2012 values). Among patients who developed mCRPC, mean costs increased from \$US2622 PPPM 12 months before diagnosis of metastasis to \$US13,291 during the month of metastasis (peak cost) and were significantly higher than costs for controls thereafter. Medical resource utilization showed a similar pattern. This study did not include the use of oral medications; however, the pattern of medical resource use and associated costs is similar to that seen in our study, with peak costs occurring during the month of mCRPC diagnosis and remaining higher than before the development of metastatic disease.

The presence of bone metastases has been shown to be associated with longer hospital stays and increased overall hospital-based treatment costs in patients with prostate cancer. In a US hospital claims database study [10], men with bone metastases had a longer length of stay (7 vs. 3 days), higher inpatient hospitalization costs (\$US14,145 
vs. 11,944; year 2010 values), and higher costs per hospital encounter (\$US9728 vs. 7405), driven largely by longer hospitalizations and inpatient pharmacy costs. Although the prostate cancer cohorts in these previous studies differed from our cohort of patients with nmCRPC who progressed to mCRPC, taken as a whole, they confirm that disease progression/metastatic disease substantially increases HCRU and costs of management of prostate cancer.

Our results also indicate that the lack of standard of care in the treatment of nmCRPC presents challenges in its management in clinical practice, as illustrated by the high number of agents used in this patient population. Also, a substantial number of patients with nmCRPC appeared to be managed with agents approved only in the metastatic setting during the time period of our study. Any off-label utilization represented herein may result in placing patients at risk for adverse events and diminished quality of life in a setting where the benefits are uncertain. Additionally, we found some chemotherapy use in the nonmetastatic setting. Finally, the time period for this study encompassed 2009 to 2015; however, in 2018, apalutamide and enzalutamide were approved for the treatment of nmCRPC $[16,17]$. The effect of these approvals on the data and results of this study are unknown, although some off-label use of enzalutamide was captured in this report. This is an important area for future research. Taken together, the significant difference in cost and resource use between $\mathrm{nmCRPC}$ and $\mathrm{mCRPC}$ highlights an opportunity for the healthcare system to reduce overall cost and resource use through optimal and standardized management of patients with nmCRPC, such that by delaying metastases, some of the costs and resource utilization during management of mCRPC can be avoided or reduced.

Some study limitations should be noted. Research conducted with claims data has inherent limitations, including possible coding errors, lack of information on over-thecounter medication or treatments obtained outside of an insurance setting, and lack of detailed clinical information (e.g., disease severity) and reasons underlying choice and provision of treatment. The MarketScan ${ }^{\circledR}$ claims databases are based on a large convenience sample, which may bias findings or affect their generalizability to other populations [13]. However, given that the average age of patients in this study was 72 years and most patients $(71 \%)$ had Medicare coverage, generalizability to the Medicare population seems reasonable.

In addition, as the index date of diagnosis of metastatic disease was defined by the date of first use of a metastatic diagnosis code, this may have allowed for misclassification of treatments in the nonmetastatic setting that were actually used to treat metastatic disease. However, a rise in cost was noted at that index diagnosis date, and this continued throughout the follow-up period. Cost and HCRU estimation limitations exist because of the follow-up period of 6 months and use of this period to estimate annualized results. The estimation of these results may be biased by the high cost and resource utilization associated with diagnosis and early treatment interventions in the first 6 months of disease. Extended follow-up in future studies is needed for corroboration of these results.

Next, this study had a relatively small sample size. During the accrual process, the largest reason for exclusion was lack of continuous enrollment in the pre- and post-index periods to ensure accurate and complete data; however, these robust criteria may have affected generalizability of the results by lowering the sample size.

The patients with nmCRPC included in the study were identified based on evidence of surgical or medical castration with subsequent progression to metastatic disease and allowing for a reasonable timeframe prior to metastatic diagnosis based on previous publications related to the natural history of the disease [14]. As metastases can develop as progression from both an nmCRPC and a hormone-sensitive state, we attempted to minimize misclassification by first capturing the diagnosis of metastases and then including only patients who had undergone surgical or medical castration. However, the diagnosis of castrate-resistant disease is typically defined as the presence of rising prostate-specific antigen in the setting of castrate levels of testosterone, which is a level of clinical detail that cannot be captured in a claims database review. Additionally, while $99.62 \%$ of our patient population were medically castrated at baseline (and for this we required combined androgen blockade with the use of one additional ADT agent plus an LHRH agent), this strategy at the time of data collection could have also been used in patients with hormone-sensitive prostate cancer [18, 19]. Regarding combined androgen blockade, the heterogeneity of prostate cancer, including how progression through the continuum of the disease occurs, can tend toward varied provider practices; therefore, the timing of dual androgen blockade may not have a consistent direct correlation with the patient disease status at that time point. However, the small sample size precluded the use of any further sensitivity analyses to ensure inclusion of only CRPC in the nonmetastatic phase. A similar algorithm used to identify patients in this population has been previously published [20], but accurate and consistent identification of patients via claims databases remains a challenge. It is imperative that research focusing on patients with nmCRPC continues to develop.

Within the nmCRPC subtype, not all patients warrant immediate treatment, but the literature is evolving to show that high-risk patients have a disease course with poor outcomes, so optimal identification and management of these patients is beneficial both clinically and for HCRU [21]. 


\section{Conclusions}

In this retrospective review, we employed an algorithm utilizing date of diagnosis of metastatic disease and prescribing patterns to identify patients with $\mathrm{mCRPC}$ from a health plan perspective. Following progression to $\mathrm{MCRPC}$, annualized all-cause HCRU and costs increased significantly. Average yearly HCRU nearly doubled and mean annual pharmacy costs had the highest proportional increase, of more than fivefold after metastasis diagnosis.

While there are noted limitations to the current study, it provides hypothesis-generating information to continue research in this area, specifically in patients with nmCRPC at high risk for early metastases. As new agents are approved in this space, there is an opportunity to delay time to development of metastatic disease and the subsequent significant increase in HCRU and cost. Our data show that delaying time to progression may be associated with decreased HCRU and costs. With the current clinical study landscape and recent drug approvals for nmCRPC, the treatment paradigm is shifting, and further studies are warranted to confirm the beneficial effect of delaying patient progression on cost and HCRU in this patient population.

Acknowledgements The authors acknowledge Adriana Valderrama, $\mathrm{PhD}, \mathrm{MBA}$, a former Bayer employee, for her support in conducting this study.

Author Contributions SA, SB, and LW developed the study design and protocol. KT led the data collection process. SA, SB, LW, KT, SS, and NS were involved in the data analysis, manuscript preparation, and manuscript review process.

Data Availability The data that support the findings of this study are available from Truven Health Analytics MarketScan ${ }^{\circledR}$, but restrictions apply to the availability of these data, which were used under license for the current study and so are not publicly available. However, data are available from the authors upon reasonable request and with permission of Truven Health Analytics MarketScan ${ }^{\circledR}$.

\section{Compliance with Ethical Standards}

Funding This study was funded by Bayer HealthCare Pharmaceuticals.

Conflict of interest SA, SB, LW, and SS are employees of Bayer HealthCare Pharmaceuticals. KT was an employee of Bayer HealthCare Pharmaceuticals at the time the study was conducted. NS is an employee of Carolina Urologic Research Institute and served as a paid consultant to Bayer.

Ethical approval Ethical or institutional review board approval was not obtained as this retrospective claims study was completed using already available data.

Informed consent Informed consent was not necessary as data sources utilized in this retrospective review were fully compliant with the Health Insurance Portability and Accountability Act of 1996 in relation to the use of deidentified patient data.
Open Access This article is distributed under the terms of the Creative Commons Attribution-NonCommercial 4.0 International License (http://creativecommons.org/licenses/by-nc/4.0/), which permits any noncommercial use, distribution, and reproduction in any medium, provided you give appropriate credit to the original author(s) and the source, provide a link to the Creative Commons license, and indicate if changes were made.

\section{References}

1. National Cancer Institute. Cancer stat facts: prostate cancer. https ://seer.cancer.gov/statfacts/html/prost.html. Accessed $26 \mathrm{Apr}$ 2018.

2. Velcheti V, Kamik S, Bardot S, Prakash O. Pathogenesis of prostate cancer: lessons from basic research. Oschner J. 2008;8:213-8.

3. Kirby M, Hirst C, Crawford ED. Characterising the castrationresistant prostate cancer population: a systematic review. Int J Clin Pract. 2011;65(11):1180-92.

4. Smith MR, Saad F, Chowdhury S, et al. Apalutamide treatment and metastasis-free survival in prostate cancer. N Engl J Med. 2018;378(15):1408-18.

5. Steinburn CN, Fizazi K, Saad F, et al. Prostate specific antigen response in men with nmCRPC (M0 CRPC) treated with enzalutamide. Results from PROSPER. Presented at the European Association of Urology; March 16-20, 2018, Copenhagen.

6. Fizazi K, Shore ND, Tammela TLJ, et al. ARAMIS trial: Efficacy and safety of ODM-201 in men with high-risk nonmetastatic castration-resistant prostate cancer. J Clin Oncol. 2016;34(15_suppl).

7. Mariotto AB, Yabroff KR, Shao Y, Feuer EJ, Brown ML. Projections of the cost of cancer care in the United States: 2010-2020. J Natl Cancer Inst. 2011;103:117-28.

8. Alemayehu B, Buysman E, Parry D, et al. Economic burden and healthcare utilization associated with castration-resistant prostate cancer in a commercial and Medicare Advantage US population. J Med Econ. 2010;13(2):351-61.

9. Li TT, Shore ND, Mehra M, et al. Impact of subsequent metastases on costs and medical resource use for prostate cancer patients initially diagnosed with localized disease. Cancer. 2017;123(18):3591-601.

10. Seal B, Sullivan SD, Ramsey SD, et al. Comparing hospital-based resource utilization and costs for prostate cancer patients with and without bone metastases. Appl Health Econ Health Policy. 2014;12(5):547-57.

11. Gorritz M, Thompson SF, Lee YC, et al. A novel algorithm using claims data to identify patients with castration-resistant prostate cancer (CRPC). Value Health. 2013;16:A427.

12. Flaig TW, Potluri RC, Ng Y, et al. Treatment evolution for metastatic castration-resistant prostate cancer with recent introduction of novel agents: retrospective analysis of real-world data. Cancer Med. 2016;5(2):182-91.

13. Hansen L. The Truven Health MarketScan ${ }^{\circledR}$ Databases for life sciences researchers. Ann Arbor: Truven Health Analytics; 2017.

14. Sartor O, Eisenberger M, Kattan M, Tombal B, Lecouvet F. Unmet needs in the prediction and detection of metastases in prostate cancer. Oncologist. 2013;18:549-57.

15. Klabunde CN, Potosky AL, Legler JM, et al. Development of a comorbidity index using physician claims data. J Clin Epidemiol. 2000;53:1258-67.

16. Apalutamide [package insert]. Horsham: Janssen Products, LP; 2018.

17. Enzalutamide [package insert]. Northbrook: Astellas Pharma US, Inc.; 2018. 
18. Mohler J, Bahnson RR, Boston B, et al. Prostate cancer. J Natl Compr Cancer Netw. 2010;8(2):162-200.

19. Mohler JL, Kantoff PW, Armstrong AJ, et al. Prostate cancer, version 2.2014. J Natl Compr Cancer Netw. 2014;12(5):686-718.

20. Wu B, Li S, Song J, et al. Annual cost of care for castrationresistant prostate cancer in U.S. commercial and Medicare supplement plans. Presented at the Academy of Managed Care Pharmacy Nexus; October 22-25, 2018, Orlando.
21. Li S, Ding Z, Lin JH, et al. Prostate-specific antigen (PSA) measurements' association with metastasis and mortality in patients with nmCRPC (non-metastatic castration resistant prostate cancer). J Clin Oncol. 2018;36(15 supplement):e17058. 